\title{
Predictability of Motor Outcome According to the Time of Motor Evoked Potentials From the Onset of Stroke in Patients With Putaminal Hemorrhage
}

\author{
Yong Min Kwon, MD, Sung Ho Jang, MD, Jung Won Lee, MD
}

Department of Physical Medicine and Rehabilitation, Yeungnam University College of Medicine, Daegu, Korea

\begin{abstract}
Objective To determine the predictability of motor evoked potentials (MEP) in patients with putaminal hemorrhage (PH) according to the time of MEP from the onset of stroke.

Methods Sixty consecutive patients with PH from January 2006 to November 2013 were retrospectively reviewed. Motor function of affected extremities was measured at onset time and at six months after the onset. Patients were classified into two groups according to the time of MEP from the onset of stroke: early MEP group (within 15 days from onset) and late MEP group (16-30 days from onset). Patients were also classified into two groups according to the presence of MEP on the affected abductor pollicis brevis (APB): MEP (+) group-patients (showing MEP in the affected APB) and MEP (-) group-patients (no MEP in the affected APB). Motor outcome was compared between the two early and late MEP groups or between the presence and absence of MEP in the affected APB groups.

Results For patients with MEP (+), a larger portion in the late MEP group showed good prognosis compared to the early MEP group (late MEP, $94.4 \%$; early MEP, 80\%). In contrast, in patients with MEP (-), a larger portion of patients in the late MEP group showed bad prognosis compared to the early MEP group (late MEP, 80\%; early MEP, 71.4\%). No significant improvement of MI between MEP (+) and MEP (-) was observed when MEP was performed early or late.

Conclusion Our results revealed that the predictability of motor outcome might be better if MEP is performed late compared to that when MEP is performed early in patients with $\mathrm{PH}$.
\end{abstract}

Keywords Transcranial magnetic stimulation, Stroke, Recovery of function, Putaminal hemorrhage, Motor evoked potentials 


\section{INTRODUCTION}

Motor weakness is one of the most serious disabling sequelae of stroke. It occurs in more than $50 \%$ of stroke patients [1]. Accurate prediction of motor outcome in stroke patients is important because it could provide useful information for clinicians to set specific rehabilitation strategies and to predict final motor outcomes. Among various neural tracts for motor function in the human brain, corticospinal tract (CST) is the most important neural tract [2-6]. Several evaluation tools have been used to predict motor outcome in stroke patients by assessing the state of CST, including conventional brain magnetic resonance imaging [7], motor evoked potentials (MEP) $[8,9]$, and diffuse tensor imaging (DTI) [10].

Transcranial magnetic stimulation (TMS) stimulates corticospinal neurons or interneurons synapsing on corticospinal neurons originating from the motor cortex. TMS can assess the state of CST by the presence of MEP or by analyzing the characteristics of MEP $[11,12]$. MEP has been widely used in the prediction of motor outcome in stroke patients $[8,9,13-16]$. However, MEP has limitation because it is possible to have false negative results due to excessive high threshold at early stage of stroke [13]. This limitation suggests that MEP might differ according to time of MEP from the onset of stroke [17]. The predictability of motor outcome in stroke patients can also differ according to the time of MEP from the onset of stroke. However, no study on this topic has been reported. We hypothesized that the predictability of MEP for motor outcome would be better in a late stage than in an early stage of stroke patients. The objective of this study was to compare the predictability of MEP in patients with putaminal hemorrhage ( $\mathrm{PH})$ according to the time of MEP from the onset of stroke.

\section{MATERIALS AND METHODS}

\section{Subjects}

We retrospectively reviewed consecutive patients with $\mathrm{PH}$ who had been admitted for rehabilitation in the Department of Rehabilitation \& Physical Medicine of Yeungnam University Hospital from January 2006 to November 2013. Sixty consecutive patients ( 39 males and 21 females with mean age of $56.06 \pm 10.97$ years) were recruited according to the following inclusion criteria: 1 ) first ever stroke, 2) age between 31 and 75 years, 3) MEP performed within 30 days from onset, 4) spontaneous intracerebral hemorrhage centered on the unilateral putamen without intra-ventricular hemorrhage confirmed by a neuroradiologist, 5) absence of serious medical complications, such as pneumonia and cardiac problems during the experimental period, and 6) no severe cognitive problems (Mini-Mental State Examination $>24$ ), no apraxia using ideomotor apraxia test [18], no somatosensory problems using two point discrimination, monofilament discrimination, and no seizure history. According to the time of MEP, patients were classified into two groups: early MEP group (within 15 days from onset) and late MEP group (16-30 days from onset). The study protocol was approved by the local ethics committee (IRB Protocol No. YUH-14-0425-D7).

\section{Clinical evaluation}

Motricity Index (MI) was measured at the onset of stroke and at six months after the stroke. MI was used to assess motor impairment in patients who have had a stroke with a minimum score of 0 and a maximum score of 100 . The reliability and validity of MI has well established [19]. Evaluations of clinical data and TMS were performed by different physicians. Based on prognosis, patients were classified into two groups: 1) good prognosis group with MI $\geq 75$ at six months after the onset and 2) poor prognosis group with $\mathrm{MI}<75$ at six months after the onset. The cutoff value of MI score at 75 was selected because affected extremities with such MI scores have been demonstrated to be able to sufficiently recover to have the ability to move against gravity [20]. Hemorrhage volume was calculated as $1 / 2 \times$ long diameter $\times$ short diameter $\times$ thickness of the high-density area on computerized tomography scan [21].

\section{TMS protocol}

TMS was performed at $17.78 \pm 4.95$ days after stroke using a Magstim 200 magnetic stimulator with a $70 \mathrm{~mm}$ (mean diameter) butterfly coil (Novametrix Inc., Wallingford, CT, USA). A cloth marked with spacing $1 \mathrm{~cm}$ apart and Cz-referenced to the intersection of midsagittal and interaural lines was placed on the scalp. The intersection of the wings (center of the coil) was applied tangentially to the scalp while the handgrips were placed so that they were parallel to the midsagittal line facing the back. Mag- 
netic stimulation was performed with excitation threshold (ET) plus $20 \%$ output. MEPs were obtained from both abductor pollicis brevis muscles (APB) in a relaxed state. Each site was stimulated three times at $1 \mathrm{~cm}$ intervals from which the shortest latency and the average of the peak-to-peak amplitudes were adopted. The site where the ET was the lowest, latency was the shortest, and average amplitude was the largest was determined as the optimal scalp site. Patients were classified into two groups according to the presence of MEP on the affected APB: MEP (+) group (patients who showed MEP in the affected APB) and MEP (-) group (patients who did not show MEP in the affected APB).

\section{Statistical analysis}

Chi-square and independent $\mathrm{t}$ tests were performed to determine the differences between two groups in terms of sex, age, lesion side, days to MEP, and hemorrhage volume. Mann-Whitney test was used to compare MI scores between at onset and at six months after onset or changes of MI score for six months of the two groups. Chi-square test was performed to compare the distribution of prognosis between early MEP and late MEP groups. SPSS ver. 17.0 software (SPSS Inc., Chicago, IL, USA) was used for all statistical analyses. Statistical significance was considered when p-value was less than 0.05 .

\section{RESULTS}

\section{Demographic and clinical data}

Demographic data of the 60 patients used in this study are summarized in Table 1. According to the time of MEP, the early MEP group had 22 patients and the late MEP group had 38 patients. In the early MEP group, 15 of the 22 patients $(68.2 \%)$ were MEP (+) and seven of the 22 patients $(31.8 \%)$ were MEP (-). In the late MEP group, 18 of the 38 patients (47.4\%) were MEP (+) and 20 of the 38 patients (52.6\%) were MEP (-). Time of MEP performed from onset was 13.0 \pm 2.20 days in the early MEP group and 20.94 \pm 3.70 days in the late MEP group. No significant ( $p>0.05$ ) differences in age, sex distribution, lesion side, or hemorrhage volume was observed between the early MEP group and the late MEP group or between the MEP (+) group and the MEP (-) group. However, significant $(\mathrm{p}<0.05)$ difference in the time of MEP performed was observed between the early MEP group and the late MEP group.

\section{Motor function in the early MEP group and late MEP group}

MI scores at onset and at six months after the onset in all subgroups are summarized in Table 2. At onset, no significant difference of MI score was observed in all subgroups. At 6 months after the onset, significant $(\mathrm{p}<0.05)$ difference was observed between MEP (+) and MEP (-) group in patients with early MEP or late MEP but no significant difference was observed between early MEP and late MEP group in patients with MEP $(+)$ or MEP $(-)$ group. In patients with MEP $(+)$, significant $(\mathrm{p}<0.05)$ difference was observed in the change of MI score for six months between the early MEP group and the late MEP group. In patients of the late MEP group, significant

Table 1. Demographic data for patients in early MEP and late MEP groups

\begin{tabular}{lccccccc}
\hline \multirow{2}{*}{ Characteristic } & \multicolumn{3}{c}{ Early MEP } & & \multicolumn{3}{c}{ Late MEP } \\
\cline { 2 - 3 } \cline { 7 - 8 } & MEP (+) & MEP (-) & Total & & MEP (+) & MEP (-) & Total \\
\hline Sex (male:female) & $15(12: 3)$ & $7(3: 4)$ & $22(15: 7)$ & & $18(11: 7)$ & $20(13: 7)$ & $38(24: 14)$ \\
Age (yr) & $57.80 \pm 10.83$ & $55.57 \pm 11.49$ & $57.09 \pm 10.81$ & & $58.50 \pm 11.28$ & $51.90 \pm 10.04$ & $55.03 \pm 11.02$ \\
Hemorrhage volume (mL) & $33.34 \pm 16.97$ & $37.53 \pm 10.11$ & $34.68 \pm 15.00$ & & $35.91 \pm 32.24$ & $43.99 \pm 23.70$ & $40.16 \pm 27.98$ \\
Lesion side & & & & & & & \\
$\quad$ Right & 8 & 4 & 12 & & 13 & 8 & 21 \\
$\quad$ Left & 7 & 3 & 10 & & 5 & 12 & 17 \\
Days to MEP & $13.00 \pm 2.20$ & $12.57 \pm 2.07$ & $12.86 \pm 2.12$ & & $20.94 \pm 3.70$ & $20.35 \pm 3.80$ & $20.63 \pm 3.72$ \\
\hline
\end{tabular}

Values are presented as mean \pm standard deviation or number of patients.

MEP, motor evoked potentials; MEP (+), patients who showed MEP in the affected abductor pollicis brevis; MEP (-), patients who did not show MEP in the affected abductor pollicis brevis; early MEP, performed within 15 days from the onset; late MEP, performed at 16-30 days after the onset. 
Table 2. Changes in motor function in the early MEP and the late MEP groups

\begin{tabular}{lccccc}
\hline \multirow{2}{*}{ Motricity Index } & \multicolumn{2}{c}{ Early MEP } & \multicolumn{2}{c}{ Late MEP } \\
\cline { 2 - 3 } \cline { 5 - 6 } & MEP (+) & MEP (-) & & MEP (+) & MEP (-) \\
\hline Onset & $36.07 \pm 25.60$ & $12.43 \pm 18.75$ & & $25.03 \pm 28.07$ & $14.98 \pm 20.54$ \\
6 Months & $81.67 \pm 10.87^{* \mathrm{a})}$ & $62.36 \pm 22.89$ & & $85.53 \pm 11.42^{* \mathrm{~b})}$ & $58.98 \pm 10.49$ \\
Difference & $45.6 \pm 29.14$ & $49.93 \pm 10.52$ & & $60.5 \pm 12.74^{* \mathrm{c}, \mathrm{d})}$ & $43.48 \pm 20.73$ \\
\hline
\end{tabular}

Values are presented as mean \pm standard deviation.

MEP, motor evoked potentials; MEP (+), patients who showed motor evoked potentials in the affected abductor pollicis brevis; MEP (-), patients who did not show motor evoked potentials in the affected abductor pollicis brevis; early MEP, performed within 15 days from the onset; late MEP, performed at 16-30 days after the onset.

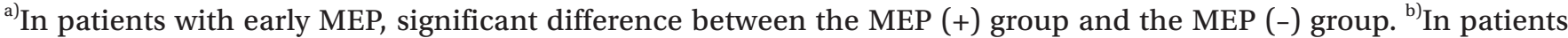
with late MEP, significant difference between the MEP (+) group and the MEP (-) group. ${ }^{c)}$ In patients with MEP (+), significant difference between the early MEP group and the late MEP group. ${ }^{\text {d) }}$ In patients who underwent late MEP, significant difference between the MEP (+) group and the MEP (-) group.

${ }^{*} \mathrm{p}<0.05$.

Table 3. Proportion of the prevalence according to the prognosis in the early MEP and the late MEP groups

\begin{tabular}{lccccccc}
\hline \multirow{2}{*}{ Motricity Index } & \multicolumn{3}{c}{ Early MEP } & & \multicolumn{3}{c}{ Late MEP } \\
\cline { 2 - 3 } \cline { 7 - 8 } & MEP (+) (n=15) & MEP (-) (n=7) & Total (n=22) & MEP (+) (n=18) & MEP (-) (n=20) & Total (n=38) \\
\hline Good prognosis & $12(80.0)$ & $2(28.6)$ & $14(63.6)$ & & $17(94.4)$ & $4(20.0)$ & $21(55.3)$ \\
Poor prognosis & $3(20.0)$ & $5(71.4)$ & $8(36.4)$ & & $1(5.6)$ & $16(80.0)$ & $17(44.7)$ \\
\hline
\end{tabular}

Values are presented as number of patients $(\%)$.

MEP, motor evoked potentials; MEP (+), patients who showed motor evoked potentials in the affected abductor pollicis brevis; MEP (-), patients who did not show motor evoked potentials in the affected abductor pollicis brevis; early MEP, performed within 15 days from the onset; late MEP, performed at 16-30 days after the onset.

$(\mathrm{p}<0.05)$ difference in change of MI score for six months was observed between the MEP (+) group and the MEP $(-)$ group. However, no significant $(\mathrm{p}>0.05)$ difference in change of MI score was observed between the early MEP group and the late MEP group in the MEP (-) group or between MEP (+) group and MEP (-) group in the early MEP group.

The clinical classification as good prognosis group $(\mathrm{MI} \geq 75)$ or poor prognosis group $(\mathrm{MI}<75)$ according to MI score at six months after stroke onset is summarized in Table 3. In the early MEP group, 14 of the 22 patients (63.6\%) belonged to the good prognosis group. The remaining 8 patients $(36.4 \%)$ belonged to the poor prognosis group. By contrast, in the late MEP group, 21 of the 38 patients (55.3\%) belonged to the good prognosis group. The remaining 17 (44.7\%) belonged to the poor prognosis group. In the early MEP group, $12(80.0 \%)$ of the 15 patients of the MEP (+) group showed good prognosis whereas five $(71.4 \%)$ of the 7 patients of the MEP (-) group showed poor prognosis. In the late MEP group, 17
(94.4\%) of 18 patients of MEP (+) showed good prognosis while $16(80.0 \%)$ of 20 patients of MEP (-) showed poor prognosis. No significant difference was observed in the distribution of prognosis between the early MEP and late MEP groups.

\section{DISCUSSION}

In the current study, we hypothesized that the predictability of MEP for motor outcome would be better in late stage than early stage of stroke patients. Thus, we compared the predictability of motor outcome in patients with $\mathrm{PH}$ according to the time when MEP was performed (early TMS, late TMS) retrospectively. We observed the following results: 1 ) for patients with MEP (+), in the late MEP group, a larger portion of patients showed good recovery compared to that in the early MEP group (late MEP, 94.4\%; early MEP, 80.0\%); 2) for patients with MEP $(-)$, in the late MEP group, a larger portion of patients had bad prognosis compared to that in the early MEP group 
(late MEP, 80.0\%; early MEP, 71.4\%); 3) No significant improvement of MI between MEP (+) and MEP (-) was observed when MEP was performed early or late. Therefore, the predictability of motor outcome in patients with $\mathrm{PH}$ when MEP was performed late (16-30 days from onset) was better than that when MEP was performed early (onset-15 days). Such results could be due to the following reasons. First, it might be due to perihematomal edema. A recent study reported that perihematomal edema could peak during the first week and continue for two weeks from onset in patients with intracerebral hemorrhage [22]. Therefore, more perihematomal edema for 15 days from stroke onset in the early MEP group might have increased the ET of MEP, consequently causing false negative results of MEP. Second, it might be due to the recovery of an injured CST with the passage of time [23,24]. Surviving penumbra around the hematoma may offer opportunities for secondary perihematomal neuronal reorganization [25-27]. Therefore, the recovered CST after injury could have resulted in more MEP (+) in the late MEP group than that in the early MEP group.

Since the introduction of MEP, many studies have reported its prediction for motor outcome in stroke patients $[8,9,13-16]$. In these studies, MEP was performed between one day and two months after the onset of stroke [8,9,13-16]. A few MEP studies have reported that absence of MEP in the early stage of stroke is unreliable for predicting of motor outcome [13,14,28]. In 1994, Arac et al. [28] performed MEP within the first week from onset in stroke patients and found that absence of MEPs obtained during the first week did not necessarily indicate poor recovery at three and six months later. In 1996, Catano et al. [13] found that ET was increased at seven days after the onset of stroke. However, ET was constantly lower at 30 days and 90 days after the onset of stroke in patients who recovered than in those who did not recover. In 2009, Pizzi et al. [14] found that an absence of MEP performed in acute post-stroke phase had a high rate of false negativity in regard to both motor outcome and functional outcome. Our results are in consistent with the results of these studies $[13,14,28]$.

Many studies using MEP have reported its predictability for motor outcome in stroke patients [8,9,13-16]. However, no study has reported the predictability of motor outcome according to the time of MEP after the onset of stroke. To the best of our knowledge, one study reported the optimal timing of DTI in stroke patients regarding other evaluation tools for predicting motor outcome. In 2012, Kwon et al. [20] compared the prediction of motor outcome according to time of DTI in 71 patients with corona radiate infarct and found that the predictability of motor outcome was better during the late two weeks than during the early two weeks after the onset of stroke. Although the evaluation tool is different, the result of Kwon's study is compatible with the result of our study. Our study is the first MEP study to investigate predictability of motor outcome according to the time of MEP in stroke patients.

There are some limitations of this study. First, we used MI score including upper and lower extremity power although MEPs were obtained from APB muscle. In addition, we classified patients into subgroups only according to the presence of MEP. Thus, outcome of MI might be affected by latency and amplitude of MEP in patient with MEP (+). Another limitation is that this study was conducted retrospectively. We could not confirm the predictability of motor outcome with the passage of time. In addition, we could not divide the duration in more details due to the small number of patients. Further studies including a larger number of patients with a prospective design should be performed in order to determine the optimal timing of MEP for more accurate prediction of motor outcome in stroke patients.

In conclusion, we found that the predictability of motor outcome might be better when MEP is performed late (16-30 days from onset) than that when MEP is performed early (onset-15 days) in patients with PH. Further studies are needed for other stroke pathologies to overcome the limitation of MEP.

\section{CONFLICT OF INTEREST}

No potential conflict of interest relevant to this article was reported.

\section{ACKNOWLEDGMENTS}

This research was supported by a grant (2012R1A1A4A01001873) of the Basic Science Research Program through the National Research Foundation of Korea (NRF) funded by the Ministry of Education, Science and Technology, Republic of Korea. Kwon YM and Jang SH 
contributed equally to this study.

\section{REFERENCES}

1. Hendricks HT, van Limbeek J, Geurts AC, Zwarts MJ. Motor recovery after stroke: a systematic review of the literature. Arch Phys Med Rehabil 2002;83:1629-37.

2. Afifi AK, Bergman RA. Functional neuroanatomy: text and atlas. 2nd ed. New York: McGraw-Hill; 2005. p. 59-60.

3. Jang SH. The corticospinal tract from the viewpoint of brain rehabilitation. J Rehabil Med 2014;46:193-9.

4. Davidoff RA. The pyramidal tract. Neurology 1990; 40:332-9.

5. Jang SH. The role of the corticospinal tract in motor recovery in patients with a stroke: a review. NeuroRehabilitation 2009;24:285-90.

6. York DH. Review of descending motor pathways involved with transcranial stimulation. Neurosurgery 1987;20:70-3.

7. DeVetten G, Coutts SB, Hill MD, Goyal M, Eesa M, O'Brien B, et al. Acute corticospinal tract Wallerian degeneration is associated with stroke outcome. Stroke 2010;41:751-6.

8. Bembenek JP, Kurczych K, Karli Nski M, Czlonkowska A. The prognostic value of motor-evoked potentials in motor recovery and functional outcome after stroke: a systematic review of the literature. Funct Neurol 2012;27:79-84.

9. van Kuijk AA, Pasman JW, Hendricks HT, Zwarts MJ, Geurts AC. Predicting hand motor recovery in severe stroke: the role of motor evoked potentials in relation to early clinical assessment. Neurorehabil Neural Repair 2009;23:45-51.

10. Jang SH. Prediction of motor outcome for hemiparetic stroke patients using diffusion tensor imaging: a review. NeuroRehabilitation 2010;27:367-72.

11. Barker AT, Jalinous R, Freeston IL. Non-invasive magnetic stimulation of human motor cortex. Lancet 1985;1:1106-7.

12. Talelli P, Greenwood RJ, Rothwell JC. Arm function after stroke: neurophysiological correlates and recovery mechanisms assessed by transcranial magnetic stimulation. Clin Neurophysiol 2006;117:1641-59.

13. Catano A, Houa M, Caroyer JM, Ducarne H, Noel P. Magnetic transcranial stimulation in acute stroke: early excitation threshold and functional prognosis. Electroencephalogr Clin Neurophysiol 1996;101:233-9.

14. Pizzi A, Carrai R, Falsini C, Martini M, Verdesca S, Grippo A. Prognostic value of motor evoked potentials in motor function recovery of upper limb after stroke. J Rehabil Med 2009;41:654-60.

15. Rapisarda G, Bastings E, de Noordhout AM, Pennisi G, Delwaide PJ. Can motor recovery in stroke patients be predicted by early transcranial magnetic stimulation? Stroke 1996;27:2191-6.

16. Timmerhuis TP, Hageman G, Oosterloo SJ, Rozeboom AR. The prognostic value of cortical magnetic stimulation in acute middle cerebral artery infarction compared to other parameters. Clin Neurol Neurosurg 1996;98:231-6.

17. Vang C, Dunbabin D, Kilpatrick D. Correlation between functional and electrophysiological recovery in acute ischemic stroke. Stroke 1999;30:2126-30.

18. Butler JA. How comparable are tests of apraxia? Clin Rehabil 2002;16:389-98.

19. Demeurisse G, Demol O, Robaye E. Motor evaluation in vascular hemiplegia. Eur Neurol 1980;19:382-9.

20. Kwon YH, Jeoung YJ, Lee J, Son SM, Kim S, Kim C, et al. Predictability of motor outcome according to the time of diffusion tensor imaging in patients with cerebral infarct. Neuroradiology 2012;54:691-7.

21. Kothari RU, Brott T, Broderick JP, Barsan WG, Sauerbeck LR, Zuccarello M, et al. The ABCs of measuring intracerebral hemorrhage volumes. Stroke 1996;27:1304-5.

22. Inaji M, Tomita H, Tone O, Tamaki M, Suzuki R, Ohno K. Chronological changes of perihematomal edema of human intracerebral hematoma. Acta Neurochir Suppl 2003;86:445-8.

23. Jang SH, Byun WM, Han BS, Park HJ, Bai D, Ahn YH, et al. Recovery of a partially damaged corticospinal tract in a patient with intracerebral hemorrhage: a diffusion tensor image study. Restor Neurol Neurosci 2006;24:25-9.

24. Yang DS, Kim DS, Kim YH, Jang SH. Demonstration of recovery of a severely damaged corticospinal tract: a diffusion tensor tractography and transcranial magnetic stimulation follow-up study. J Comput Assist Tomogr 2008;32:418-20.

25. Furlan M, Marchal G, Viader F, Derlon JM, Baron JC. Spontaneous neurological recovery after stroke 
and the fate of the ischemic penumbra. Ann Neurol 1996;40:216-26.

26. Witte OW. Lesion-induced plasticity as a potential mechanism for recovery and rehabilitative training. Curr Opin Neurol 1998;11:655-62.

27. Cramer SC. Repairing the human brain after stroke:
I. Mechanisms of spontaneous recovery. Ann Neurol 2008;63:272-87.

28. Arac N, Sagduyu A, Binai S, Ertekin C. Prognostic value of transcranial magnetic stimulation in acute stroke. Stroke 1994;25:2183-6. 